\title{
Diagnóstico post-mortem de infarto agudo do miocárdio em pacientes com insuficiência respiratória aguda - análise demográfica, etiológica e histológica pulmonar
}

\author{
Post-mortem diagnosis of acute myocardial infarction in \\ patients with acute respiratory failure - demographic, \\ etiologic and histological pulmonary analysis
}

\author{
Aline Domingos Pinto Ruppert, Marcella Soares Pincelli, André Domingos Pinto \\ Ruppert, Alexandre de Matos Soeiro, Carlos V. Serrano Junior, Vera Luiza Capelozzi
}

Ruppert ADP, Pincelli MS, Ruppert ADP, Soeiro AM, Serrano Junior CV, Capelozzi VL. Diagnóstico post-mortem de infarto agudo do miocárdio em pacientes com insuficiência respiratória aguda - análise demográfica, etiológica e histológica pulmonar/ Post-mortem diagnosis of acute myocardial infarction in patients with acute respiratory failure - demographic, etiologic and histological pulmonary analysis. Rev Med (São Paulo). 2011 jan.-mar.;90(1):29-35.

RESUMO: Introdução: A insuficiência respiratória aguda (IRA) está presente em $5 \%$ dos pacientes com infarto agudo do miocárdio (IAM) e é associada à mortalidade de $20 \%$ a $30 \%$ nesses pacientes. Não está claro o papel da inflamação relacionada ao edema agudo de pulmão (EAP) na gênese da IRA pós IAM. Objetivos: Descrever os dados demográficos, etiológicos e os achados histológicos pulmonares em autópsias realizadas uentre 1990 e 2008, de pacientes que morreram por IRA, sem diagnóstico in-vivo de IAM. Métodos: Este estudo considerou 4223 autópsias de pacientes que morreram de IRA nos quais só foi definida postmortem sua causa de morte. O diagnóstico de IAM foi feito por autópsia em 218 (4,63\%) pacientes, dos quais foram obtidos: idade, sexo e principais doenças associadas. Os achados pulmonares histológicos foram classificados em: dano alveolar difuso (DAD), edema agudo de pulmão (EAP), hemorragia alveolar (HA) e pneumonia intersticial linfo-plasmocitária (PILP). A probabilidade de IAM desenvolver determinado tipo de achado histopatológico pulmonar foi calculada por regressão logística. Resultados: Foram observados 147 homens, e a mediana de idade foi 64 anos. A análise histopatológica pulmonar mostrou, em ordem decrescente: EAP (72,9\%), DAD, PILP e HIA. Broncopneumonia bacteriana esteve presente em $11,9 \%$, hipertensão arterial sistêmica em $10,1 \%$, miocardiopatia dilatada em $6,9 \%$, tromboembolismo pulmonar em $6,0 \%$, cardiomiopatia hipertrófica em $4,6 \%$, doença pulmonar obstrutiva crônica em $3,7 \%$ e diabetes mellitus em $3,7 \%$ dos pacientes. A análise multivariada demonstrou associação significativamente positiva de IAM com EAP e DAD. Conclusões: Pela primeira vez na literatura, demonstramos, por meio de autópsias, que em pacientes com IRA que evoluem à óbito sem diagnóstico estabelecido, IAM esteve presente em aproximadamente $5 \%$ dos casos. Nós observamos importante componente inflamatório na histologia pulmonar, nunca antes sugerido.

DESCRITORES: Insuficiência respiratória/mortalidade; Infarto do miocárdio/mortalidade; Infarto do miocárdio/diagnóstico; Inflamação; Edema pulmonar; Autopsia.
ABSTRACT: Introduction: Acute respiratory failure (ARF) is present in $5 \%$ of the patients with acute myocardial infarction (AMI) and is responsible for $20 \%$ to $30 \%$ of the mortality post-AMI. It is unclear the role of inflammation correlated with pulmonary edema (PE) as a cause of ARF post-AMI. Objectives: Describe the demographic, etiologic data and histological pulmonary findings in autopsies of patients dead due to ARF with non-diagnosis AMI during life between 1,990 and 2,008. Methods: This study considers 4,223 autopsies of patients who died of ARF without cause of death related during life. The diagnosis of AMI was performed in 218 (4.63\%) patients, and were obtained: age, sex and major associated diseases. Pulmonary histopathology was categorized as: diffuse alveolar damage (DAD); pulmonary edema (PE); alveolar hemorrhage (AH);and lympho-plamacytic interstitial pneumonia (LPIP). Odds ratio of AMI developing specific histopathology was determined by logistic regression. Results: Were observed 147 men and mean age was 64 years. Pulmonary histopathology showed, in descending order: PE (72.9\%), DAD, LPIP and HA. Bacterial bronchopneumonia was present in $11.9 \%$, systemic arterial hypertension in $10.1 \%$, dilated cardiomyopathy in $6.9 \%$, pulmonary embolism in $6.0 \%$, hypertrophic cardiomyopathy in $4.6 \%$, chronic obstructive pulmonary disease in $3.7 \%$ and diabetes mellitus in $3.7 \%$ of patients. Multivariate analysis demonstrated significantly positive association of IAM with PE and with DAD. Conclusions: For the first time we demonstrated that in autopsies of patients with ARF as cause of death, the diagnosis of AMI was present in about $5 \%$. We observed important inflammatory response in pulmonary histology as never suggested before.

KEYWORDS: Respiratory insufficiency/mortality; Myocardial infarction/mortality; Myocardial infarction/diagnosis; Inflammation; Pulmonary edema; Autopsy.

COMU 2010 - Prêmio Oswaldo Cruz - Área Clínica.

Faculdade de Medicina da Universidade de São Paulo.

Endereço para correspondência: Vera L. Capelozzi. Depto. Patologia da FMUSP. Av. Dr. Arnaldo, 455 - São Paulo, SP, Brasil. 


\section{INTRODUÇÃO}

insuficiência respiratória aguda (IRA)
é uma das principais causas de morte
em pacientes acometidos por diversos tipos de comorbidades ${ }^{1-4}$. A mortalidade tem sido relatada como maior que $40-50 \%$, especialmente em pacientes que apresentam infiltração difusa à radiografia de tórax e desenvolvimento de síndrome do desconforto respiratório agudo ${ }^{1-3}$. Achados clínicos e radiológicos de IRA são inespecíficos ${ }^{5-7}$ e a pronta investigação e o diagnóstico precoce da doença de base são essenciais na melhora da sobrevida dos pacientes ${ }^{8-10}$. Além disso, a complexidade de apresentações clínicas faz do diagnóstico etiológico da IRA um constante desafio para o médico ${ }^{8-10}$.

Reconhecidamente, o infarto agudo do miocárdio (IAM) pode apresentar-se na forma de IRA em aproximadamente $17 \%$ dos pacientes e está associado a um prognóstico muito reduzido a curto e longo prazos ${ }^{11-15}$. A mortalidade intra-hospitalar e após um ano de seguimento pode ser observada, respectivamente, em $17 \%$ e $35 \%$ dos $\operatorname{casos}^{11,16}$. O diagnóstico exato e imediato do IAM em pacientes com apresentação inespecífica de IRA no serviço de emergência pode melhorar a sobrevida ${ }^{13,16,17}$. O papel da inflamação em IRA após IAM tem sido estudado e parece ser tão importante quanto o aumento das pressões de enchimento vasculares ${ }^{17}$. Nenhuma informação foi descrita sobre achados pulmonares relacionados à resposta inflamatória pulmonar nestes pacientes.

Nesse contexto, desenvolvemos um estudo retrospectivo de 4710 autópsias em pacientes com IRA como causa de morte e IAM não diagnosticado in vivo, a fim de melhor descrever os dados demográficos, etiológicos e os respectivos achados histológicos pulmonares e doenças associadas.

\section{MATERIAIS E MÉTODOS}

\section{Autópsias}

O presente estudo foi desenvolvido em um centro de saúde de complexidade terciária. Entre 1990 e 2008, 26.560 autópsias foram revisadas (média anual de 1.889 autópsias). Nós consideramos $4.710(17,7 \%)$ pacientes, cuja causa de morte foi IRA sem diagnóstico específico relatado em vida. Nestes, o diagnóstico de IAM foi realizado durante a autópsia em 218 (4,63\%) casos. A análise histológica pulmonar foi realizada em 210 casos de IAM. Em oito pacientes, os achados histológicos pulmonares não puderam ser revisados porque o tecido pulmonar não se encontrava disponível para consulta.

Neste estudo foram considerados todos os diagnósticos macroscópicos e microscópicos de morte presentes nas autópsias e também, os prontuários médicos dos pacientes incluídos. IRA foi definida como $\mathrm{PaO} 2<60 \mathrm{mmHg}$ ou $\mathrm{PaCO} 2>$ $50 \mathrm{mmHg}$ com $\mathrm{Ph}<7,30$, em ar ambiente ${ }^{1-3}$. Foram excluídos pacientes com menos de um ano de idade e indivíduos sem diagnóstico de IRA e IAM.

A extensão e a localização do IAM puderam, na maior parte dos casos, ser mais adequadamente avaliadas através de cortes transversais que abrangeram as cavidades ventriculares. Investigaramse as primeiras alterações macroscópicas em casos com óbito entre 12 e 24 horas, procurando-se por áreas afetadas de coloração relativamente mais escura, levemente intumescidas, com ou sem pontos hemorrágicos, sem limites precisos.

Do ponto de vista microscópico, as alterações foram investigadas para a presença de "necrose de coagulação" em todos os casos com mais de 6 horas de óbito. Na periferia da zona infartada, investigou-se a presença de miocitólise (ou degeneração vacuolar). Inflamação aguda, com aporte de neutrófilos, perda das estriações celulares, cariorrexis, e tecido de granulação ricamente vascularizado, foram também incluídos no exame histológico cardíaco.

Foram obtidos dados referentes à idade, sexo e principais doenças associadas (determinadas na autópsia) para cada paciente.

As informações clínicas dos pacientes selecionados neste estudo foram coletadas após a obtenção de termo de consentimento livre e esclarecido por um membro da família e após a aprovação pelo comitê de ética e pesquisa.

Após uma completa revisão, os achados histopatológicos pulmonares foram classificados em:

Dano alveolar difuso (DAD): difuso envolvimento e aparecimento temporal uniforme de colapso alveolar, membranas hialinas, fibrose obliterativa, formação de neo-septos e fibrose moderadamente organizada;

$>$ Edema agudo de pulmão (EAP): acúmulo de fluido proteináceo nos espaços alveolares, dando aspecto de granulação e coagulação rósea dentro de tais espaços;

$>$ Hemorragia alveolar (HA): presença de 
sangue nos espaços alveolares;

$>$ Pneumonia intersticial linfo-plasmocítica (PILP): septos alveolares alargados e edemaciados, geralmente acompanhados por infiltrado mononuclear inflamatório composto por linfócitos, histiócitos, células plasmocitárias e neutrófilos.

Todos os pulmões foram analisados por microscopia, mesmo quando os laudos indicavam os diagnósticos e achados do paciente. Por no mínimo quatro semanas, os pulmões foram fixados em formalina $10 \%$. Examinamos um mínimo de cinco lâminas por pulmão (totalizando dez lâminas por caso). As secções de tecido embebidas em parafina foram coradas com hematoxilina e eosina. Para documentar a presença e a distribuição do largo espectro de agentes infecciosos para os quais a população é suscetível, nós preparamos uma variedade de corantes especiais (coloração ácido periódico-Schiff, análise imuno-histoquímica, fluorescência, Ziehl-Neelsen, Gram, Mucicarmine e prata metanamina de Gomori) para os cortes de tecido selecionados. Broncopneumonia bacteriana (BCP) foi definida como a presença de consolidação celular com acúmulo de leucócitos polimorfonucleares nos bronquíolos e alvéolos adjacentes. Para o diagnóstico de citomegalovírus e pneumonia fúngica, a evidência do envolvimento histológico dos pulmões foi requerida com ou sem cultura dos tecidos. Sepse grave e/ou choque séptico foi definido como sepse com presença de disfunção de órgãos ou diagnóstico clínico de hipotensão arterial, que podem ou não ser responsível à ressuscitação volêmica agressiva. O diagnóstico de infecção por Mycobacterium tuberculosis e infecção por micobactérias atípicas foi confirmada usando fluorescência, técnica ZiehlNeelsen e cultura Lowenstein-Jensen. Os métodos proporcional e bioquímico foram usados para a identificação de todas as culturas positivas.

\section{Análise estatística}

A análise descritiva dos dados coletados incluíram mediana, valores mínimos e máximos. A probabilidade (odds ratio) de pacientes com IAM desenvolverem determinado padrão histopatológico pulmonar foi determinada por regressão logística. As variáveis utilizadas incluíram as principais doenças e/ou comorbidades encontradas: BCP, hipertensão arterial sistêmica, cardiomiopatia dilatada, tromboembolismo pulmonar, cardiomiopatia hipertrófica, doença pulmonar obstrutiva crônica, diabetes mellitus, sepse grave e/ou choque séptico e acidente vascular cerebral. Todos os procedimentos estatísticos foram executados utilizando o software SPSS v10.0 statistical. Foi considerado estatisticamente significativo $p<0,05$.

\section{RESULTADOS}

IAM não diagnosticado em vida foi descrito em 218 autópsias $(4,63 \%)$ dos pacientes com IRA entre 1990 e 2008. A idade dos pacientes variou de 16 a 90 anos (mediana de 64$)$. Um total de $147(67,4 \%)$ homens e $71(32,6 \%)$ mulheres foram incluídos no estudo. Os achados demográficos estão expostos na Tabela 1.

TABELA 1. Achados demográficos em autópsias de pacientes com IRA e IAM

\begin{tabular}{cccc}
\hline & \multicolumn{2}{c}{ Sexo } & \\
\cline { 2 - 3 } $\begin{array}{c}\text { Faixa etá- } \\
\text { ria (anos) }\end{array}$ & $\begin{array}{c}\text { Mascu- } \\
\text { lino }\end{array}$ & $\begin{array}{c}\text { Femini- } \\
\text { no }\end{array}$ & Total \\
\hline 1 to 50 & 28 & 17 & $45(20,6 \%)$ \\
51 to 80 & 106 & 45 & $\begin{array}{c}151 \\
(69,3 \%)\end{array}$ \\
$>80$ & 13 & 9 & $22(10,1 \%)$ \\
Total & 147 & 71 & 218 \\
\hline
\end{tabular}

Foi observada a associação de somente um diagnóstico somado a IAM em $46(21,1 \%)$ casos, dois diagnósticos em $16(7,3 \%)$, três em $16(7,3 \%)$ e quatro em 8 (3,7\%) casos. Em 1322 (60,6\%) pacientes, IAM foi a única doença relatada nas autópsias. Um total de 158 doenças e/ou comorbidades foi observado além de IAM. A maioria dos diagnósticos associados à pacientes com IRA e IAM está exposta na Tabela 2.

TABELA 2. Principais diagnósticos associados à autópsias de pacientes com IRA e IAM

\begin{tabular}{lc}
\hline \multicolumn{1}{c}{ Doenças associadas } & $\begin{array}{c}\text { Número total } \\
\text { (\% de casos) }\end{array}$ \\
\hline BCP & $26(11,9 \%)$ \\
HAS & $22(10,1 \%)$ \\
Cardiomiopatia dilatada & $15(6,9 \%)$ \\
TEP & $13(6,0 \%)$ \\
Cardiomiopatia hipertrófica & $10(4,6 \%)$ \\
DPOC & $8(3,7 \%)$ \\
Diabetes mellitus & $8(3,7 \%)$ \\
Sepse grave e/ou choque & $7(3,2 \%)$ \\
séptico & $6(2,8 \%)$ \\
AVC &
\end{tabular}

${ }^{*} \mathrm{BCP}=$ broncopneumonia; $\mathrm{HAS}=$ hipertensão arterial sistêmica; TEP = tromboembolismo pulmonar; $\mathrm{DPOC}=$ doença pulmonar obstrutiva crônica; AVC = acidente vascular cerebral. 
Ruppert et al. Diagnóstico post-mortem de infarto agudo do miocárdio.

BCP esteve presente em $11,9 \%$ dos pacientes (26 casos) e foi a mais freqüente complicação pulmonar encontrada durante a autópsia. Hipertensão arterial sistêmica foi a segunda mais freqüente, tendo sido observada em $10,1 \%$ dos pacientes (22 casos), seguida de cardiomiopatia dilatada em 6,9\% (15 casos), tromboembolismo pulmonar em 6,0\% (13 casos), cardiomiopatia hipertrófica em 4,6\% (10 casos), doença pulmonar obstrutiva crônica em $3,7 \%$ ( 8 casos), diabetes mellitus in 3,7\% (8 casos), sepse grave e/ou choque séptico em $3,2 \%$ ( 7 casos) e acidente vascular cerebral em 2.8\% (6 casos) dos pacientes. Outros diagnósticos com menor prevalência somaram $27,2 \%$ do total de doenças encontradas.

A análise histopatológica pulmonar mostrou EAP em 72,9\% (159) dos pacientes, DAD em 17,4\% (38), PILP em 4,1\% (9) and HA em 1,8\% (4).

A análise multivariada com associação estatística entre IAM e diferentes achados histopatológicos pode ser verificada na Tabela 3.

TABELA 3. Análise multivariada com associação estatística entre IAM e diferentes achados histopatológicos pulmonares

\begin{tabular}{cccccccccccccc}
\hline \multicolumn{1}{c}{ DAD } & EAP & HA & PILP & & & & & & & \\
\hline & OR & $p^{* *}$ & IC $95 \%$ & OR & $p^{* *}$ & IC $95 \%$ & OR & $p^{* *}$ & IC $95 \%$ & OR & $p^{* *}$ & IC $95 \%$ \\
IAM & 3,14 & $<0,001$ & $2,18-4,54$ & 6,19 & $<0,001$ & $4,50-8,52$ & 5,67 & 0,001 & $2,08-15,44$ & 4,94 & NS & $0,71-6,32$
\end{tabular}

${ }^{*} \mathrm{DAD}=$ dano alveolar difuso; $\mathrm{EAP}=$ edema agudo de pulmão; $\mathrm{HA}=$ hemorragia alveolar; PILP = pneumonia intersticial linfo-plasmocítica; $\mathrm{IAM}=$ infarto agudo do miocárdio; $\mathrm{OR}=$ odds ratio; IC = intervalo de confiança; NS = não significativo.

${ }^{* *} p<0,05$

\section{DISCUSSÃO}

Trata-se do principal estudo em autópsia a incluir informações demográficas, diagnósticos etiológicos e os respectivos achados histopatológicos pulmonares em pacientes com IRA e IAM (não diagnosticado in-vivo). IRA foi descrita em 4.710 pacientes, e IAM esteve presente em 4,68\% (218) dos pacientes. Em 132 (60,6\%) pacientes IAM foi o único diagnóstico relatado na autópsia. Conforme já referida, BCP esteve presente em 11,9\% (26 casos) e hipertensão arterial sistêmica em 10,1\% (22) dos pacientes. A histopatologia pulmonar mostrou EAP em $72,9 \%$ e DAD em $17,4 \%$ dos pacientes. A análise multivariada mostrou associação significativa de IAM com EAP e com DAD.

O tratamento precoce e correto de IRA permanece um importante problema no manejo de pacientes em estado crítico. Apesar dos recentes avanços tecnológicos em diagnóstico, a autópsia se mantém uma ferramenta essencial para identificação e compreensão de doenças. Estudos em autópsia têm mostrado significativas diferenças entre os achados da autópsia e os diagnósticos clínicos antemortem ${ }^{18-25}$. Tal discordância pode variar de 10 a $90 \%$, dependendo da doença e da população envolvida ${ }^{5-10,19-21,26-32}$. Perkins et al. ${ }^{19}$ mostrou que em pacientes que morreram em unidades de terapia intensiva, o diagnóstico correto in-vivo não foi realizado em $39 \%$ dos casos. IAM foi o principal diagnóstico não obtido em vida, e esteve presente em aproximadamente $10 \%$ desses casos. Além disso, em quase todos os pacientes estudados com IAM, o diagnóstico clinico pré-morte foi BCP. Estas discrepâncias poderiam ser atribuídas a diferentes manifestações clínicas de uma única doença e à pobre qualidade do atendimento médico ${ }^{5}$.

Nesse estudo, observamos uma prevalência de aproximadamente $5 \%$ de IAM não diagnosticados em pacientes com IRA. Outros autores revelaram dados semelhantes ${ }^{19,22}$. Uma comparação publicada em 2007 entre diagnósticos clínicos e em autópsias, em um hospital de cardiologia, relatou $5 \%$ de IAM não diagnosticados no total de mortes por todas as causas. A taxa de concordância entre autópsias e diagnósticos dados em vida de IAM foi $71 \%^{22}$.

A maioria dos pacientes analisados foi do sexo masculino (67\%) e a mediana da idade foi de 64 anos. Na literatura tem sido classicamente descrita a alta ocorrência de equivalente isquêmico como dispnéia em mulheres e pacientes mais idosos ${ }^{17}$. A maioria dos estudos mostrou uma mediana de idade ente 69 e 80 anos em pacientes com EAP pós$\mathrm{IAM}^{11-16}$. Entretanto, observamos um maior número de diagnósticos em homens, de idade não tão avançada como seria esperado, talvez devido à associação entre IRA e outras causas de morte, por vezes não consideradas em outros estudos. Flutowski et al. ${ }^{16}$ 
relatou uma mediana de idade em pacientes com EAP pós-IAM de 70 anos. Além disso, apresentaram uma prevalência de mulheres em $54 \%$ dos casos $^{16}$.

Notamos que em 132 (60,6\%) pacientes, IAM foi o único diagnóstico relatado nas autópsias. Um grande número de doenças e/ou comorbidades (158 casos) foi observado além do IAM em pacientes com IRA. BCP esteve presente em $11,9 \%$ dos pacientes e foi a mais freqüente complicação pulmonar encontrada durante a autópsia, seguida de hipertensão arterial sistêmica (10,1\%). Freqüentemente, pacientes com IRA têm BCP como causa inicial de doença pulmonar, mas algumas vezes BCP aparece como uma complicação de outras patologias, principalmente em pacientes imunocomprometidos e intubados ${ }^{33-36}$.

Cardiomiopatia dilatada, tromboembolismo pulmonar, cardiomiopatia hipertrófica, doença pulmonar obstrutiva crônica, diabetes mellitus, sepse grave e/ou choque séptico e acidente vascular cerebral também estiveram presentes em grande número de casos. A maior prevalência $(39,4 \%$ dos casos) de um ou mais diagnósticos mostra a necessidade de diferentes estratégicas terapêuticas para estes pacientes, conferindo prognóstico reservado 4 ,13,19,21-24. Um estudo publicado em 2008, ao analisar o uso contínuo de pressão positiva em pacientes com EAP secundário a IAM, mostrou mortalidade hospitalar de $11 \%$, relatando que todos os pacientes mortos tinham diagnósticos associados como sepse grave, BCP, acidente vascular cerebral e tromboembolismo pulmonar, semelhante às doenças listadas em nosso estudo ${ }^{12}$.

Conforme esperado, EAP foi o padrão mais comumente observado ( $72,9 \%$ dos casos) e também foi o mais específico achado associado ao IAM, como descrito previamente em outros estudos ${ }^{4,16,17}$. IAM tem sido descrito como o mais importante fator etiológico associado com o desenvolvimento de EAP em pacientes hospitalizados ${ }^{16}$. IAM é a mais freqüente causa de insuficiência cardíaca e EAP, principalmente devido à conseqüente elevação da pressão diastólica no ventrículo esquerdo e da pressão veno-capilar pulmonar, e também devido à redistribuição de fluido dos capilares pulmonares aos espaços intersticial e alveolar ${ }^{16,17}$.

Surpreendentemente, DAD esteve associado também a IAM. Não encontramos tal associação na literatura e nenhuma informação semelhante foi encontrada em pacientes com IAM. Isto indica que uma importante resposta inflamatória pode estar presente na IRA pós-IAM, conforme sugerido por outros autores, especificamente relacionada ao choque cardiogênico ${ }^{11,17,37-40}$. Há um interesse crescente no papel de mediadores inflamatórios em IAM. A lesão miocárdica é, em parte, o resultado da ativação do sistema inflamatório, com produção e liberação de citocinas pró-inflamatórias, ativação do sistema complemento, produção de auto-anticorpos, hiperexpressão da maioria das moléculas do complexo de histocompatibilidade e expressão de moléculas de adesão que podem perpetuar o estado inflamatório ${ }^{40}$. Neste contexto, as citocinas mais estudadas são o fator de necrose tumoral e a interleucina-6, mas outros, como proteína- $C$ reativa, selectinas e endotelina-1, relatadas em choque cardiogênico pós-IAM ${ }^{11,37,40}$. Alguns estudos sugerem que a interleucina-6 é mediadora da ativação endotelial em vasos pulmonares ${ }^{11}$. A endotelina-1 poderia aumentar a permeabilidade vascular por indução da produção de moléculas de adesão leucocitárias e pela conseqüente ativação de neutrófilos, amplificando a resposta inflamatória nos pulmões ${ }^{38}$. Grandes estudos investigacionais são necessários para um melhor entendimento da ativação de citocinas inflamatórias em território pulmonar pós-IAM. Este conhecimento pode melhorar a sobrevida de pacientes na medida em que viabiliza medidas preventivas, diagnóstico precoce e terapêutico direcionada ${ }^{40}$.

Hemorragia alveolar também esteve associada ao IAM. Nós imaginamos, porém, que sua associação com IAM não foi tão relevante devido ao pequeno número de pacientes (4 casos).

\section{Limitações}

Primeiramente, trata-se de um estudo retrospectivo sobre laudos de autópsias, nas quais a qualidade de informações pode apresentar limitações. Outra limitação de nosso estudo está relacionada à variabilidade do observador, mesmo levando em conta que, em nossa instituição, todas as autópsias são feitas por um patologista residente e supervisionadas por um médico assistente da área que também checa a análise histológica na preparação do laudo final.

Diferentes opiniões podem ser obtidas de diferentes observadores. Ademais, a acurácia dos achados de autópsia também dependem do interesse e da habilidade do patologista.

\section{CONCLUSÕES}

Apesar dos avanços tecnológicos em diagnóstico, a autópsia continua a se destacar como importante ferramenta de complementação para a identificação e o entendimento de doenças em pacientes com IRA. Pela primeira vez, mostramos que em autópsias de pacientes com IRA como causa de morte, o diagnóstico de IAM esteve presente em 5\% dos casos. BCP foi o diagnóstico mais comum dentre 
os pacientes com IRA e IAM. O padrão histopatológico pulmonar mais prevalente foi EAP, mas observamos importante resposta inflamatória na histopatologia pulmonar, como nunca antes sugerido.

\section{REFERÊNCIAS}

1. Barbas CS, Capelozzi VL, Hoelz C, Magaldi RB, Souza R, Sandeville ML, et al. Impacto de biópsia pulmonar a céu aberto na insuficiência respiratória aguda refratária. J Bras Pneumol. 2006;32(5):41823.

2. Milberg JA, Davis DR, Steinberg KP, Hudson LD. Improved survival of patients with acute respiratory distress syndrome (ARDS): 1983-1993. JAMA. 1995;273(4):306-9.

3. Behrendt CE. Acute respiratory failure in the United States: incidence and 31-day survival. Chest. 2000;118(4):1100.

4. Soeiro AM, Parra ER, Canzian M, Farhat C, Capelozzi VL. Alterações histopatológicas pulmonares em pacientes com insuficiência respiratória aguda: um estudo em autópsias. J Bras Pneumol. 2008;34(2):6773.

5. Castellanos Ortega A, Ortiz Melón F, García Fuentes M, Prieto Valderrey F, Santidrián Miguel JP, Mazorra Macho $F$. The evaluation of autopsy in the pediatric intensive unit. An Esp Pediatr. 1997;46(3):224-8.

6. Fernandez-Segoviano $P$, Lázaro A, Esteban A, Rubio JM, Iruretagoyena JR. Autopsy as quality assurance in the intensive care unit. Crit Care Med. 1988;16(7):683-5.

7. Stevanovic G, Tucakovic G, Dotlic R, Kanjuh V. Correlation of clinical diagnoses with autopsy findings: a retrospective study of 2,145 consecutive autopsies. Hum Pathol. 1986;17(12):1225-30.

8. Blosser SA, Zimmerman HE, Stauffer JL. Do autopsies of critically ill patients reveal important findings that were clinically undetected? Crit Care Med. 1998;26(8):1332-6.

9. Kumar P, Taxy J, Angst DB, Mangurten HH. Autopsies in children: are they still useful? Arch Pediatr Adolesc Med. 1998;152(6):558-63.

10. Mort TC, Yeston NS. The relationship of pre mortem diagnoses and post mortem findings in a surgical intensive care unit. Crit Care Med. 1999;27(2):299303.

11. Olga M, Cotter G, Kaluski E, Brill A, Blatt A, Krakover $\mathrm{R}$, et al. Comparison of inflammatory and neurohormonal activation in cardiogenic pulmonary edema secondary to ischemic versus nonisquemic causes. Am J Cardiol. 2003;92(15):222-6.

12. Consentini R, Aliberti S, Bignamini A, Piffer F, Brambilla AM. Mortality in acute cardiogenic pulmonary edema treated with continuous positive airway pressure. Intensive Care Med. 2009;35:299305.

13. Parakh K, Thombs BD, Bhat U, Fauerbach JA, Bush
Novos estudos são necessários para elucidação completa dos mecanismos fisiopatológicos envolvidos no IAM relacionado ao desenvolvimento da IRA.

DE, Roy C, et al. Long-term significance of killip class and left ventricular systolic dysfunction. The American J Med. 2008;121:1015-8.

14. Lewis EF, Velazquez EJ, Solomon SD, Hellkamp AS, McMurray JJV, Mathias J, et al. Predictors of the first heart failure hospitalization in patients who are stable survivors of myocardial infarction complicated by pulmonary congestion and/or left ventricular dysfunction: a VALIANT study. Eur Heart J. 2008;29:748-56.

15. Ware LB, Matthay MA. Acute pulmonary edema. N Engl J Med. 2005;353:2788-96.

16. Fiutowski M, Waszyrowski T, Krzeminska-Pakula M, Kasprzak JD. Pulmonary edema prognostic score predicts in-hospital mortality risk in patients with acute cardiogenic pulmonary edema. Heart Lung. 2008;37:46-53.

17. Hochman JS. Cardiogenic shock complicating acute myocardial infarction: expanding the paradigm. Circulation. 2003;107:2998-3002.

18. Grade MHC, Zucoloto S, Kajiwara JK, Fernandes MTP, Couto LGF, Garcia SB.Trends of accuracy of clinical diagnoses of the basic cause of death in a university hospital. J Clin Pathol. 2004;57:369-73.

19. Perkins GD, McAuley DF, Davies S, Gao F. Discrepamcies between clinical and postmortem diagnoses in critically ill patients: an observational study. Critical Care. 2003;7:R129-R132.

20. Shojania KG, Burton EC, McDonald KM, Goldman $L$. Changes in rates of autopsy-detected diagnosis errors over time. JAMA. 2003;289(21):2849-56.

21. Roulson J, Benbow EW, Hasleton PS. Discrepancies between clinical and autopsy diagnosis and the value of post mortem histology; a meta-analysis and review. Histopathology. 2005;47:551-9.

22. Saad R, Yamada AT, Rosa FHFP. Comparison between clinical and autopsy diagnoses in a cardiology hospital. Heart. 2007;93:1414-9.

23. Afessa B, Green W, Chiao J, Frederick W. Pulmonary complications of HIV infection: autopsy findings. Chest. 1998;113:1225-9.

24. Lanjewar DN, Duggal R. Pulmonary pathology in patients with AIDS: an autopsy study from Mumbai. HIV Med. 2001;2:266-71.

25. Canzian M, Soeiro AM, Taga, MFL, Farhat C, Barbas CSV, Capelozzi VL. Semiquantitative assessment of surgical lung biopsy: predictive value and impact on survival of patients with diffuse pulmonary infiltrate. Clinics. 2007;62:23.

26. Canzian M, Soeiro AM, Taga MF, Barbas CS, 
Capelozzi VL. Correlation between surgical lung biopsy and autopsy findings and clinical data in patients with diffuse pulmonary infiltrates and acute respiratory failure. Clinics. 2006;61(5):425-32.

27. Gerain J, Sculier JP, Malengreaux A, Rykaert C, Thémelin L. Causes of deaths in an oncologic intensive care unit: a clinical and pathological study of 34 autopsies. Eur J Cancer. 1990;26(3):377-81.

28. Tóth T, Szöts I, Juhász M, Benedek G. The importance of pulmonary complications as a cause of death in surgical patients. Int Surg. 1984;69(1):35-7.

29. Motsay GJ, Lillehei RC. Acute respiratory distress syndrome in adults. Definition, etiology and treatment. Int Surg. 1973;58(5):304-7.

30. Friederici $\mathrm{HH}$, Sebastian M. Autopsies in a modern teaching hospital. A review of 2,537 cases. Arch Pathol Lab Med. 1984;108(6):518-21.

31. Thornton CM, O'Hara MD. A regional audit of perinatal and infant autopsies in Northern Ireland. Br J Obstet Gynaecol. 1998;105(1):18-23.

32. Demling $\mathrm{RH}$, Nerlich $M$. Acute respiratory failure. Surg Clin North Am. 1983;63(2):337-55.

33. Parambil JG, Myers JL, Aubry MC, Ryu JH. Causes and prognosis of diffuse alveolar damage diagnosed on surgical lung biopsy. Chest. 2007;132:50-7.

34. Pinheiro BV, Muraoka FS, Assis RVC, Lamin R, Pinto SPS, Ribeiro Júnior PJ, et al. Accuracy of clinical diagnosis of acute respiratory distress syndrome in comparison with autopsy findings. J Bras Pneumol. 2007;33(4):423-8.

35. Rao VK, Ritter J, Kollef MH. Utilty of transbronchial biopsy in patients with acute respiratory failure - a postmortem study. Chest. 1998;114:549-55.

36. Gross JS, Neufeld RR, Libow LS, Gerber I, Rodstein M. Autopsy study of the elderly institutionalized patient. Review of 234 autopsies. Arch Intern Med. 1998;148:173-6.

37. Geppert A, Huber K. Inflammation and cardiovascular diseases: lessons that can be learned for the patient with cardiogenic shock in the intensive care unit. Curr Opin Crit Care. 2004;10:347-53.

38. Magder S, Cernacek P. Role of endothelins in septic, cardiogenic, and hemorrhagic shock. Can J Pharmacol. 2003;81:635-43.

39. Bodi V, Sanchis J, Nunez J, Mainar L, Minana G, Benet I, et al. Uncontrolled immune response in acute myocardial infarction: Unraveling the thread. Am Heart J. 2008;156:1065-73.

40. Chen D, Assad-Kottner C, Orrego C, Torre-Amione G. Cytokines and acute heart failure. Crit Care Med. 2008;36(1):S9-S16.

Artigo premiado COMU: 18-23/10/10. 